\title{
FIXED-POINT-LIKE THEOREMS ON SUBSPACES
}

\author{
PHILIPPE BICH AND BERNARD CORNET
}

Received 8 June 2004

We prove a fixed-point-like theorem for multivalued mappings defined on the finite Cartesian product of Grassmannian manifolds and convex sets. Our result generalizes two different kinds of theorems: the fixed-point-like theorem by Hirsch et al. (1990) or Husseini et al. (1990) and the fixed-point theorem by Gale and Mas-Colell (1975) (which generalizes Kakutani's theorem (1941)).

\section{Introduction}

In this paper, we prove a fixed-point-like theorem for multivalued mappings defined on the finite Cartesian product of Grassmannian manifolds and convex sets. Let $k$ be an integer and let $V$ be a Euclidean space such that $0 \leq k \leq \operatorname{dim} V$, then the $k$-Grassmannian manifold of $V$, denoted $G^{k}(V)$, is the set of all the $k$-dimensional subspaces of $V$. The set $G^{k}(V)$ is a smooth compact manifold but, in general, it does not satisfy properties such as convexity or acyclicity and its Euler characteristic may be null. This prevents the use of classical fixed-point theorems as Brouwer's [2], Kakutani's [14], or EilenbergMontgomery's theorem [7].

Our main result generalizes two different kinds of theorems: the fixed-point-like theorem by Hirsch et al. [11] or Husseini et al. [13] and the fixed-point theorem by Gale and Mas-Colell [8] (which generalizes Kakutani's theorem [14]). As in [11, 13], we will mainly use techniques from degree theory. As a consequence of our main result, we first deduce the standard fixed-point theorems when the variable is in a convex domain (such as Brouwer and Kakutani's theorem) and second Borsuk-Ulam's theorem.

The main result of this paper is directly motivated by the existence problem of equilibria in economic models with incomplete markets; in [1], it is used to extend the classical existence result by Duffie and Shafer [6] to the nontransitive case.

The paper is organized as follows. The main result is stated in Section 2 together with some direct consequences of it, namely, the results by Hirsch et al. [11], Gale and MasColell [8] and Borsuk-Ulam's theorem. The proof of the main result is given in Section 3 
and the appendix recalls the main properties of the Grassmannian manifold, used in this paper.

\section{Statement of the results}

2.1. Preliminaries. A correspondence $\Phi$ from a set $X$ to a set $Y$ is a map from $X$ to the set of all the subsets of $Y$, and the graph of $\Phi$, denoted $\mathrm{G}(\Phi)$, is defined by $\mathrm{G}(\Phi)=\{(x, y) \in$ $X \times Y \mid y \in \Phi(x)\}$. A mapping $\varphi: X \rightarrow Y$ is said to be a selection of $\Phi$ if $\varphi(x) \in \Phi(x)$ for all $x \in X$. If $A$ is a subset of $X$, we let $\Phi(A)=\bigcup_{x \in A} \Phi(x)$, and the restriction of $\Phi$ to $A$, denoted $\left.\Phi\right|_{A}$, is the correspondence from $A$ to $Y$ defined by $\left.\Phi\right|_{A}(x)=\Phi(x)$ if $x \in A$. If $X$ and $Y$ are topological spaces, the correspondence $\Phi$ is said to be lower semicontinuous (l.s.c.) (resp., upper semicontinuous (u.s.c.)) if for every open set $U \subset Y$, the set $\{x \in X \mid$ $\Phi(x) \cap U \neq \varnothing\}$ is open in $X$ (resp., the set $\{x \in X \mid \Phi(x) \subset U\}$ is open in $X$ and, for every $x \in X, \Phi(x)$ is compact).

If $x=\left(x_{1}, \ldots, x_{n}\right)$ and $y=\left(y_{1}, \ldots, y_{n}\right)$ belong to $\mathbb{R}^{n}$, we denote by $x \cdot y=\sum_{i=1}^{n} x_{i} y_{i}$ the scalar product of $\mathbb{R}^{n},\|x\|=\sqrt{x \cdot x}$ the Euclidian norm. If $x \in \mathbb{R}^{n}$ and $r \in \mathbb{R}_{+}$, we let $B(x, r)=\left\{y \in \mathbb{R}^{n} \mid\|x-y\|<r\right\}$ and $\bar{B}(x, r)=\left\{y \in \mathbb{R}^{n} \mid\|x-y\| \leq r\right\}$. If $E$ is a vector subspace of $\mathbb{R}^{n}$, we denote by $E^{\perp}=\left\{u \in \mathbb{R}^{n} \mid \forall x \in E, x \cdot u=0\right\}$ the orthogonal space to $E$. If $u_{1}, \ldots, u_{k}$ belong to $E$, a vector space, we denote by $\operatorname{span}\left\{u_{1}, \ldots, u_{k}\right\}$ the vector subspace of $E$ spanned by $u_{1}, \ldots, u_{k}$.

Let $V$ be a Euclidean space and let $k$ be an integer such that $0 \leq k \leq \operatorname{dim} V$; we denote by $G^{k}(V)$ the set consisting of all the linear subspaces of $V$ of dimension $k$, called the $(k-)$ Grassmannian manifold of $V$. Then it is known that $G^{k}(V)$ is a smooth manifold of dimension $k(\operatorname{dim} V-k)$ and we refer to the appendix for the properties we will use hereafter, together with the precise definition of the manifold structure on $G^{k}(V)$.

2.2. The main result and some consequences. The aim of this paper is to prove the following result.

Theorem 2.1. Let $I$, $J$ be two finite disjoint sets. For every $i \in I$, let $k_{i}$ be an integer and let $V_{i}$ be a Euclidean space such that $0 \leq k_{i} \leq \operatorname{dim} V_{i}$. For every $j \in J$, let $C_{j}$ be a nonempty, convex, compact subset of a Euclidean space $V_{j}$, and let $M=\prod_{i \in I} G^{k_{i}}\left(V_{i}\right) \times \prod_{j \in J} C_{j}$.

For $i \in I$ and $k=1, \ldots, k_{i}$, let $F_{i}^{k}$ be a correspondence from $M$ to $V_{i}$ with convex values, for $j \in J$, let $F_{j}$ be a correspondence from $M$ to $C_{j}$ with convex values, and suppose that, for every $i \in I$ and $k=1, \ldots, k_{i}$ (resp., $j \in J$ ), the correspondence $F_{i}^{k}$ (resp., $F_{j}$ ) is either l.s.c or u.s.c.

Then, there exists $\bar{x}=\left(\left(\bar{x}_{i}\right)_{i \in I},\left(\bar{x}_{j}\right)_{j \in J}\right) \in M$ such that

(i) either $F_{i}^{k}(\bar{x}) \cap \bar{x}_{i} \neq \varnothing$ or $F_{i}^{k}(\bar{x})=\varnothing$ for every $i \in I$ and $k=1, \ldots, k_{i}$;

(ii) either $F_{j}(\bar{x}) \cap\left\{\bar{x}_{j}\right\} \neq \varnothing$ or $F_{j}(\bar{x})=\varnothing$ for every $j \in J$.

The proof of Theorem 2.1 is given in Section 3. A first consequence of Theorem 2.1 is the following theorem by Hirsch et al. [11].

Corollary 2.2. Let $V_{1}$ be a Euclidean space, let $k_{1}$ be an integer such that $0 \leq k_{1} \leq \operatorname{dim} V_{1}$, and for every $k=1, \ldots, k_{1}$, let $f^{k}: G^{k_{1}}\left(V_{1}\right) \rightarrow V_{1}$ be a continuous mapping. Then, there exists $\bar{x} \in G^{k_{1}}\left(V_{1}\right)$ such that for every $k=1, \ldots, k_{1}, f^{k}(\bar{x}) \in \bar{x}$. 
Proof. Take $I=\{1\}, J=\varnothing$, and $F_{1}{ }^{k}(x)=\left\{f^{k}(x)\right\}$ for every $x \in G^{k_{1}}\left(V_{1}\right)$ and for every $k=$ $1, \ldots, k_{1}$. From Theorem 2.1, there exists $\bar{x} \in M=G^{k_{1}}\left(V_{1}\right)$ such that for every $k=1, \ldots, k_{1}$, $F_{1}^{k}(\bar{x}) \cap \bar{x} \neq \varnothing$, that is, $f^{k}(\bar{x}) \in \bar{x}$.

A second consequence of Theorem 2.1 is the following generalization of Gale and MasColell's theorem [8], which is also a generalization of Kakutani's theorem. Hereafter, we use the formulation by Gourdel [9] allowing each correspondence to be either u.s.c. or 1.s.c.

Corollary 2.3. Let J be a finite set, for $j \in J$, let $C_{j}$ be a nonempty, convex, compact subset of a Euclidean space, and let $F_{j}$ be a correspondence from $M:=\prod_{j \in J} C_{j}$ to $C_{j}$ with convex values, such that the correspondence $F_{j}$ is either l.s.c or u.s.c. Then, there exists $\bar{x}=\left(\bar{x}_{j}\right)_{j \in J} \in$ $M$ such that for every $j \in J$, either $\bar{x}_{j} \in F_{j}(\bar{x})$ or $F_{j}(\bar{x})=\varnothing$.

Proof. Take $I=\varnothing$ and apply Theorem 2.1.

Remark 2.4. According to our definition, an u.s.c. correspondence has compact values and without this requirement, Theorem 2.1 may not be true, as we can see in the following counterexample. Let $M:=G^{1}\left(\mathbb{R}^{2}\right)$. Each element $D$ of $G^{1}\left(\mathbb{R}^{2}\right)$ can be written as $D_{t}=\{\lambda(\cos t, \sin t) \mid \lambda \in \mathbb{R}\}$, for some $t \in[0, \pi[$. We define the correspondence $F$ from $M$ to $\mathbb{R}^{2}$ by $F\left(D_{0}\right)=\mathbb{R} \times\{1\}$ and $F\left(D_{t}\right)=D_{t} \cap(\mathbb{R} \times\{1\})+\{(1,0)\}$ if $\left.t \in\right] 0, \pi[$. We let the reader check that for every open set $U \subset \mathbb{R}^{2}$, the set $\{x \in M \mid F(x) \subset U\}$ is open in $M$ and that $F$ has nonempty, convex (and closed) values. Yet, it is straightforward that $F(x) \cap x=\varnothing$ for every $x \in G^{1}\left(\mathbb{R}^{2}\right)$.

Another consequence of our main result is the following multivalued version of Borsuk and Ulam's theorem. We denote by $S^{n}$ the unit sphere of $\mathbb{R}^{n+1}$.

Corollary 2.5. For $k=1, \ldots, n$, let $F^{k}$ be a correspondence from $S^{n}$ to $\mathbb{R}$ with nonempty and convex values such that for every $k=1, \ldots, n, F^{k}$ is either l.s.c or u.s.c. Then, there exists $\bar{x} \in S^{n}$ such that

$$
\forall k \in\{1, \ldots, n\}, \quad F^{k}(\bar{x}) \cap F^{k}(-\bar{x}) \neq \varnothing .
$$

Proof. For every $k=1, \ldots, n$, let $\hat{F}^{k}$ be the correspondence from $S^{n}$ to $\mathbb{R}$ defined by

$$
\hat{F}^{k}(x)=\left\{u-v \mid u \in F^{k}(x), v \in F^{k}(-x)\right\} .
$$

We let the reader check that for every $k=1, \ldots, n$, the correspondence $\hat{F}^{k}$ has nonempty, convex values and that it is u.s.c. (resp., l.s.c.) if $F^{k}$ is u.s.c. (resp., l.s.c.). So, to prove Corollary 2.5, it suffices to show the existence of $\bar{x} \in S^{n}$ such that $0 \in \hat{F}^{k}(\bar{x})$ for every $k=1, \ldots, n$.

We define, for every $k=1, \ldots, n$, the correspondence $H^{k}$ from $G^{n}\left(\mathbb{R}^{n+1}\right)$ to $\mathbb{R}^{n+1}$ as follows: for every $E \in G^{n}\left(\mathbb{R}^{n+1}\right)$, we let $H^{k}(E)=\hat{F}^{k}(x) x$, where $x$ is an arbitrary element of $E^{\perp} \cap S^{n}$. The correspondence $H^{k}$ is well defined since $E^{\perp} \cap S^{n}=\{x,-x\}$ for some element $x \in S^{n}$ and since $\hat{F}^{k}(x) x=\hat{F}^{k}(-x)(-x)$. 
Take $I=\{1\}, V_{1}=\mathbb{R}^{n+1}, k_{1}=n, J=\varnothing$, and apply Theorem 2.1 to the correspondences $H^{k}$, which clearly satisfy the assumptions of Theorem 2.1 . So there exists $\bar{E} \in G^{n}\left(\mathbb{R}^{n+1}\right)$ such that $\bar{E} \cap H^{k}(\bar{E}) \neq \varnothing$ for every $k=1, \ldots, n$.

Now, if $\bar{x}$ is an arbitrary point of $\bar{E}^{\perp} \cap S^{n}$, then we have $\bar{E} \cap \hat{F}^{k}(\bar{x}) \bar{x} \neq \varnothing$; from $\bar{x} \in \bar{E}^{\perp}$ and $\bar{x} \neq 0$, we finally obtain $0 \in \hat{F}^{k}(\bar{x})$ for every $k=1, \ldots, n$, which ends the proof of Corollary 2.5 .

\section{Proof of Theorem 2.1}

The proof is given in three steps, corresponding to the following three subsections. The first step gives the proof under the additional assumptions that $J=\varnothing$ and the correspondences $F_{i}^{k}$ are single-valued. The second step only assumes in addition that $J=\varnothing$. Finally, the third step gives the proof under the assumptions of Theorem 2.1.

3.1. Proof when $J=\varnothing$ and $F_{i}^{k}$ are single-valued. We first prove Theorem 2.1 under the additional assumptions that $J=\varnothing$ and the $F_{i}^{k}$ are single-valued. This is exactly the statement below.

Theorem 3.1. Let I be a finite set and for $i \in I$, let $k_{i}$ be an integer and let $V_{i}$ be a Euclidean space such that $0 \leq k_{i} \leq \operatorname{dim} V_{i}$. Let $M:=\prod_{i \in I} G^{k_{i}}\left(V_{i}\right)$ and for $i \in I$, let $f_{i}: M \rightarrow\left(V_{i}\right)^{k_{i}}$ be a continuous mapping. Then, there exists $\bar{x}=\left(\bar{x}_{i}\right)_{i \in I} \in M$ such that

$$
\forall i \in I, \quad f_{i}(\bar{x}) \in\left(\bar{x}_{i}\right)^{k_{i}} .
$$

The proof of Theorem 3.1 is given in two steps. In the first step, we additionally assume that the mappings are smooth, and the second step gives the proof in the general case.

3.1.1. Proof of Theorem 3.1 when the $f_{i}$ are smooth. Let $M:=\prod_{i \in I} G^{k_{i}}\left(V_{i}\right)$ and define $f$ : $M \rightarrow \prod_{i \in I} V_{i}^{k_{i}}$ by

$$
f(x)=\left(\operatorname{proj}_{\left(x_{i}^{k_{i}}\right)^{\perp}} f_{i}(x)\right)_{i \in I} \text { for } x=\left(x_{i}\right)_{i \in I} \in M,
$$

and the subsets $Z, Z_{1}$, and $Z_{2}$ of $M \times \prod_{i \in I} V_{i}^{k_{i}}$ by

$$
\begin{aligned}
& Z=\left\{(x, y) \in M \times \prod_{i \in I} V_{i}^{k_{i}} \mid \forall i \in I, y_{i} \in\left(x_{i}^{k_{i}}\right)^{\perp}\right\}, \\
& Z_{1}=\left\{(x, y) \in M \times \prod_{i \in I} V_{i}^{k_{i}} \mid y=f(x)\right\}, \\
& Z_{2}=\left\{(x, y) \in M \times \prod_{i \in I} V_{i}^{k_{i}} \mid y=0\right\} .
\end{aligned}
$$

Proving Theorem 3.1 amounts to showing the existence of $\bar{x} \in M$ such that $f(\bar{x})=0$ or, equivalently, such that $Z_{1} \cap Z_{2} \neq \varnothing$. For this, we will use the following Intersection Theorem 3.2, which is a direct consequence of $\bmod 2$ intersection theory (see, e.g., [10, page 79] and [5, page 127]). 
INTERSECTION THEOREM 3.2. Let $Z$ be a smooth boundaryless manifold of dimension $2 m$ and let $Z_{1}, Z_{2}$ be two compact boundaryless submanifolds of $Z$ of dimension $m$. If $\bar{Z}_{1}$ is a compact boundaryless $m$-submanifold of $Z$ homotopic to $Z_{1}$ and if the manifolds $\bar{Z}_{1}$ and $Z_{2}$ intersect transversally in a unique point $\bar{z}$ (which means that $T_{\bar{z}} \bar{Z}_{1}+T_{\bar{z}} Z_{2}=T_{\bar{z}} Z$ ), then $Z_{1} \cap Z_{2} \neq \varnothing$.

The proof of Theorem 3.1 consists of checking that the above-defined sets $Z, Z_{1}$, and $Z_{2}$ (together with the set $\bar{Z}_{1}$ defined below) satisfy the assumptions of Intersection Theorem 3.2.

The sets $Z, Z_{1}$, and $Z_{2}$ satisfy the assumptions of Intersection Theorem 3.2. We recall that for every $i \in I, G^{k_{i}}\left(V_{i}\right)$ is a smooth, boundaryless, compact manifold of dimension $k_{i}\left(\operatorname{dim} V_{i}-k_{i}\right)$ (see Lemma A.1 in the appendix). Thus $M:=\prod_{i \in I} G^{k_{i}}\left(V_{i}\right)$ is a boundaryless, smooth, compact manifold of dimension $m=\sum_{i \in I} k_{i}\left(\operatorname{dim} V_{i}-k_{i}\right)$. Clearly $Z$ is a fiber bundle whose base space is $M$ and whose fiber at $x=\left(x_{i}\right)_{i \in I} \in M$ is the vector space $\prod_{i \in I}\left(x_{i}^{k_{i}}\right)^{\perp}$ which has the dimension of $M$. Hence, $Z$ is a smooth manifold of dimension $2 m$.

The mapping $f: M \rightarrow \prod_{i \in I} V_{i}^{k_{i}}$ is a smooth mapping from Parts (c), (d), and (e) of Lemma A. 1 in the appendix. Consequently, $Z_{1}$ is a smooth compact boundaryless submanifold of $Z$ of dimension $m$. Finally, $Z_{2}$ is clearly a smooth boundaryless compact submanifold of $Z$ of dimension $m$.

The manifold $Z_{1}$ is homotopic to the manifold $\bar{Z}_{1}$ that we now define. For every $i \in I$, let $\bar{x}_{i} \in G^{k_{i}}\left(V_{i}\right)$ and let $\left\{\bar{e}_{i}^{1}, \ldots, \bar{e}_{i}^{k_{i}}\right\}$ be an orthonormal basis of $\bar{x}_{i}$. For every $i \in I$, let $g_{i}: G^{k_{i}}\left(V_{i}\right) \rightarrow V_{i}^{k_{i}}$ and $g: M \rightarrow \prod_{i \in I} V_{i}^{k_{i}}$ be the mappings defined as follows:

$$
\begin{aligned}
\forall x_{i} \in G^{k_{i}}\left(V_{i}\right), & g_{i}\left(x_{i}\right)=\left(\operatorname{proj}_{x_{i^{\perp}}}\left(\bar{e}_{i}^{1}\right), \ldots, \operatorname{proj}_{x_{i}^{\perp}}\left(\bar{e}_{i}^{k_{i}}\right)\right) \in\left(x_{i}^{\perp}\right)^{k_{i}}=\left(x_{i}^{k_{i}}\right)^{\perp}, \\
& \forall x=\left(x_{i}\right)_{i \in I} \in M, \quad g(x)=\left(g_{i}\left(x_{i}\right)\right)_{i \in I^{\prime}}
\end{aligned}
$$

We let

$$
\bar{Z}_{1}:=\left\{(x, y) \in M \times \prod_{i \in I} V_{i}^{k_{i}} \mid y=g(x)\right\} .
$$

To show that the manifold $Z_{1}$ is homotopic to $\bar{Z}_{1}$, we let $H:[0,1] \times Z_{1} \rightarrow Z$ be the continuous mapping defined by $H(t,(x, f(x)))=(x,(1-t) f(x)+\operatorname{tg}(x))$. Then $H(0, \cdot)$ is the canonical inclusion from $Z_{1}$ to $Z$, and $H(1, \cdot)\left(Z_{1}\right)=\bar{Z}_{1}$.

The manifolds $\bar{Z}_{1}$ and $Z_{2}$ intersect transversally in a unique point. First, notice that $\bar{Z}_{1} \cap Z_{2}=\left\{(x, 0) \in M \times \prod_{i \in I} V_{i}^{k_{i}} \mid g(x)=0\right\}$ is the singleton $(\bar{x}, 0)=\left(\left(\bar{x}_{i}\right)_{i \in I}, 0\right)$. But that $\bar{Z}_{1}$ and $Z_{2}$ intersect each other transversally in $Z$ means that $T_{(\bar{x}, 0)} \bar{Z}_{1}+T_{(\bar{x}, 0)} Z_{2}=T_{(\bar{x}, 0)} Z$. Recalling that $\operatorname{dim} T_{(\bar{x}, 0)} \bar{Z}_{1}+\operatorname{dim} T_{(\bar{x}, 0)} Z_{2}=\operatorname{dim} T_{(\bar{x}, 0)} Z=2 m$, we only have to show that $T_{(\bar{x}, 0)} \bar{Z}_{1} \cap T_{(\bar{x}, 0)} Z_{2}=\{0\}$. Finally, noticing that $T_{(\bar{x}, 0)} \bar{Z}_{1}=\left\{(u, D g(\bar{x})(u)) \mid u \in T_{\bar{x}} M\right\}$ and $T_{(\bar{x}, 0)} Z_{2}=\left\{(u, 0) \mid u \in T_{\bar{x}} M\right\}$, we only have to prove that $\operatorname{Dg}(\bar{x})$ is injective, which is proved in the following lemma.

Lemma 3.3. $\operatorname{Dg}(\bar{x})$ is injective.

Proof. Recalling that for every $x=\left(x_{i}\right)_{i \in I} \in M, g(x)=\left(g_{i}\left(x_{i}\right)\right)_{i \in I}$, the mapping $\operatorname{Dg}(\bar{x})$ is injective if and only if for every $i \in I, D g_{i}\left(\bar{x}_{i}\right)$ is injective. 
So, let $i \in I$, let $(\varphi, U)$ be a local chart of $G^{k_{i}}\left(V_{i}\right)$ at $\bar{x}_{i}$, and let $\psi:\left(\bar{x}_{i}^{\perp}\right)^{k_{i}} \rightarrow G^{k_{i}}\left(V_{i}\right)$ be the inverse mapping of $\varphi: U \rightarrow\left(\bar{x}_{i}^{\perp}\right)^{k_{i}}$. From the appendix, if $\left\{\bar{e}_{1}^{1}, \ldots, \bar{e}_{i}^{k_{i}}\right\}$ is a given orthonormal basis of $\bar{x}_{i}, \psi$ can be defined by

$$
\psi\left(u^{1}, \ldots, u^{k_{i}}\right)=\operatorname{span}\left\{\bar{e}_{i}^{-1}+u^{1}, \ldots, \bar{e}_{i}^{k_{i}}+u^{k_{i}}\right\} \quad \text { for every }\left(u^{1}, \ldots, u^{k_{i}}\right) \in\left(\bar{x}_{i}^{\perp}\right)^{k_{i}} .
$$

Since the mapping $g_{i} \circ \psi$ is the local representation $g_{i}$ in the chart $(\varphi, U)$, proving that $D g_{i}\left(\bar{x}_{i}\right)$ is injective amounts to proving that $D\left(g_{i} \circ \psi\right)(0)$ is injective. This is a consequence of the following claim.

ClaIm 3.4. For all $\left(h_{1}, \ldots, h_{k_{i}}\right) \in\left(\bar{x}_{i}^{\perp}\right)^{k_{i}}, D\left(g_{i} \circ \psi\right)(0)\left(h_{1}, \ldots, h_{k_{i}}\right)=-\left(h_{1}, \ldots, h_{k_{i}}\right)$.

Proof of Claim 3.4. Let $p: V_{i} \times\left(\bar{x}_{i}^{\perp}\right)^{k_{i}} \rightarrow V_{i}$ be defined by

$$
p(y, u):=\operatorname{proj}_{\psi(u)} y .
$$

If we prove that for every $y \in V_{i}$, the derivative of the mapping $p_{y}: u \rightarrow p(y, u)$ is the linear mapping $D p_{y}(0):\left(\bar{x}_{i}^{\perp}\right)^{k_{i}} \rightarrow V_{i}$ defined by

$$
D p_{y}(0)(h)=\sum_{k=1}^{k_{i}}\left(y \cdot \bar{e}_{i}^{k}\right) h_{k}, \quad \forall h=\left(h_{1}, \ldots, h_{k_{i}}\right) \in\left(\bar{x}_{i}^{\perp}\right)^{k_{i}},
$$

then Claim 3.4 will be proved. Indeed, taking $y=\bar{e}_{i}^{k}$ for every $k=1, \ldots, k_{i}$, we would obtain $D_{\bar{e}_{i}^{k}} p(0)\left(h 1, \ldots, h_{k_{i}}\right)=h_{k}$. Thus, since $g_{i} \circ \psi(u)=\left(\bar{e}_{i}^{1}, \ldots, \bar{e}_{i}^{k_{i}}\right)-\left(p_{\bar{e}_{i}^{1}}(u), \ldots, p_{\bar{e}_{i}^{k_{i}}}(u)\right)$, it would entail Claim 3.4.

Now, for every $u=\left(u_{1}, \ldots, u_{k_{i}}\right) \in\left(\bar{x}_{i}^{\perp}\right)^{k_{i}}$, there exists $\lambda(y, u)=\left(\lambda_{k}(y, u)\right)_{k=1, \ldots, k_{i}} \in \mathbb{R}^{k_{i}}$ such that

$$
p(y, u)=\operatorname{proj}_{\psi(u)} y=\sum_{k=1}^{k_{i}} \lambda_{k}(y, u)\left(\bar{e}_{i}^{k}+u^{k}\right),
$$

with $\left(\lambda_{k}(y, u)\right)$ satisfying

$$
\left(-y+\sum_{k=1}^{k_{i}} \lambda_{k}(y, u)\left(\bar{e}_{i}^{k}+u^{k}\right)\right) \cdot\left(\bar{e}_{i}^{j}+u^{j}\right)=0 \quad \text { for every } j=1, \ldots, k_{i} .
$$

This can be equivalently rewritten as follows:

$$
\left(I_{k_{i}}+G(u)\right) \lambda(y, u)=\left(y \cdot\left(\bar{e}_{i}^{1}+u^{1}\right), \ldots, y \cdot\left(\bar{e}_{i}^{k_{i}}+u^{k_{i}}\right)\right),
$$

where $I_{k_{i}}$ is the $k_{i} \times k_{i}$ identity matrix and $G(u)$ is the $k_{i} \times k_{i}$ matrix $G(u)=$ $\left(u^{j} \cdot u^{k}\right)_{j, k=1, \ldots, k_{i}}$. Besides, for $u$ in a neighborhood $\mathcal{N}$ of 0 small enough, the matrix $\left(I_{k_{i}}+\right.$ $G(u))$ is invertible. Consequently, the mapping $\lambda(\cdot, \cdot)$ is smooth on $V \times \mathcal{N}$, which implies 
that the mapping $p(\cdot, \cdot)$ is smooth on $V \times \mathcal{N}$. Differentiating, with respect to $u$, the above equality at $u=0$, we obtain, for every $h=\left(h_{1}, \ldots, h_{k_{i}}\right) \in\left(\bar{x}_{i}^{\perp}\right)^{k_{i}}$,

$$
D G(0)(h) \lambda(y, 0)+D_{u} \lambda(y, 0)(h)=0 .
$$

But it is clear that $D G(0)=0$. Consequently, $D_{u} \lambda(y, 0)=0$.

Finally, differentiating the equality $p(y, u)=\sum_{k=1}^{k_{i}} \lambda_{k}(y, u)\left(\bar{e}_{i}^{k}+u^{k}\right)$ at $(y, 0)$, one obtains, for every $h=\left(h_{k}\right)_{k=1}^{k_{i}} \in\left(\bar{x}_{i}^{\perp}\right)^{k}$,

$$
D_{u} p(y, 0)(h)=\sum_{k=1}^{k_{i}} \lambda_{k}(y, 0) h_{k}=\sum_{k=1}^{k_{i}}\left(y \cdot \bar{e}_{i}^{k}\right) h_{k}
$$

which ends the proof of Claim 3.4.

3.1.2. Proof of Theorem 3.1 in the general case. Since $M$ is a compact manifold and $V_{i}^{k_{i}}$ is a Euclidean space, for every $i \in I$, each continuous mapping $f_{i}: M \rightarrow V_{i}^{k_{i}}$ can be approximated by a sequence of smooth mappings $f_{i}^{n}: M \rightarrow V_{i}^{k_{i}}$ converging to $f_{i}$, in the sense that $\lim _{n \rightarrow \infty}\left\|f_{i}^{n}-f_{i}\right\|_{\infty}=0$ (see, e.g., Hirsch [12]). Applying the first step to the smooth mappings $f_{i}^{n}$, we deduce the existence of $\left(x_{i}^{n}\right)_{i \in I} \in M$ such that

$$
\forall i \in I, \quad f_{i}^{n}\left(x_{i}^{n}\right) \in\left(x_{i}^{n}\right)^{k_{i}}
$$

or, equivalently,

$$
\operatorname{proj}_{\left(x_{i}^{n \perp}\right)^{k_{i}}} f_{i}\left(x_{i}^{n}\right)=0 .
$$

From the compactness of $M$, without any loss of generality, one can suppose that the sequence $\left(x_{i}^{n}\right)_{i \in I}$ converges to some element $\left(\bar{x}_{i}\right)_{i \in I} \in M$. We have

$$
\begin{aligned}
& \quad\left\|\operatorname{proj}_{\left(\bar{x}_{i}^{\perp}\right)^{k_{i}}} f_{i}\left(\bar{x}_{i}\right)-\operatorname{proj}_{\left(\bar{x}_{i}^{n \perp}\right)^{k_{i}}} f_{i}^{n}\left(x_{i}^{n}\right)\right\| \\
& \quad \leq\left\|\operatorname{proj}_{\left(\bar{x}_{i}^{\perp}\right)^{k_{i}}} f_{i}\left(\bar{x}_{i}\right)-\operatorname{proj}_{\left(\bar{x}_{i}^{n \perp}\right)^{k_{i}}} f_{i}\left(x_{i}^{n}\right)\right\|+\left\|f_{i}^{n}-f_{i}\right\|_{\infty} .
\end{aligned}
$$

Consequently, from the convergence of $f_{i}^{n}$ to $f_{i}$ and the continuity of the mapping $(u, v) \rightarrow \operatorname{proj}_{\left(u^{\perp}\right)^{k_{i}}} v$ (see Lemma A.1 in the appendix), we obtain

$$
\operatorname{proj}_{\left(\bar{x}_{i}^{\perp}\right)^{k_{i}}} f_{i}\left(\bar{x}_{i}\right)=0
$$

or, equivalently,

$$
\forall i \in I, \quad f_{i}\left(\bar{x}_{i}\right) \in\left(\left(\bar{x}_{i}^{\perp}\right)^{k_{i}}\right)^{\perp}=\left(\bar{x}_{i}\right)^{k_{i}},
$$

which ends the proof of Theorem 3.1. 
3.2. Proof of Theorem 2.1 when $J=\varnothing$. We now prove Theorem 2.1 when $J=\varnothing$. The proof rests on the following claim.

Claim 3.5. For every $i \in I$ and every $k \in\left\{1, \ldots, k_{i}\right\}$, there exists an u.s.c. correspondence $\hat{F}_{i}^{k}$ from $M$ to $V_{i}$, with nonempty convex values, such that

$$
\forall x \in M, \quad\left[F_{i}^{k}(x) \neq \varnothing\right] \Longrightarrow\left[\forall y \in \hat{F}_{i}^{k}(x), \exists \lambda \in \mathbb{R}, \lambda y \in F_{i}^{k}(x)\right] .
$$

Proof of Claim 3.5. Let $i \in I$ and $k \in\left\{1, \ldots, k_{i}\right\}$. We distinguish two cases.

Assume first that $F_{i}^{k}$ is l.s.c. Let $U_{i}^{k}=\left\{x \in M \mid F_{i}^{k}(x) \neq \varnothing\right\}$. Then $U_{i}^{k}$ is an open subset of $M$ and $\left.F_{i}^{k}\right|_{U_{i}^{k}}$ is a l.s.c. correspondence with nonempty convex values. By Michael [15], there exists a continuous selection $f_{i}^{k}$ of $\left.F_{i}^{k}\right|_{U_{i}^{k}}$, that is, $f_{i}^{k}: U_{i}^{k} \rightarrow V_{i}$ is a continuous mapping such that $f_{i}^{k}(x) \in F_{i}^{k}(x)$ for every $x \in U_{i}^{k}$. Let $B_{i}$ be the closed unit ball of $V_{i}$, and we define the correspondence $\hat{F}_{i}^{k}$ from $M$ to $B_{i}$ by $\hat{F}_{i}^{k}(x)=\left\{f_{i}^{k}(x) /\left\|f_{i}^{k}(x)\right\|\right\}$ if $x \in U_{i}^{k}$ and $f_{i}^{k}(x) \neq 0$ and $\hat{F}_{i}^{k}(x)=B_{i}$ otherwise. We let the reader check that the correspondence $\hat{F}_{i}^{k}$ satisfies the conclusion of Claim 3.5.

We now consider the case where $F_{i}^{k}$ is u.s.c. Let $U_{i}^{k}=\left\{x \in M \mid F_{i}^{k}(x) \neq \varnothing\right\}$. Then $U_{i}^{k}$ is a closed subset of $M$. By Cellina [4], one can extend $\left.F_{i}^{k}\right|_{U_{i}}$ as follows: there exists a correspondence $\hat{F}_{i}^{k}$ from $M$ to $V_{i}$ which is u.s.c., with nonempty, convex, and compact values, such that for every $x \in U_{i}^{k}, F_{i}^{k}(x)=\hat{F}_{i}^{k}(x)$.

We now come back to the proof of Theorem 2.1 when $J=\varnothing$. For every $i \in I$ and $k=1, \ldots, k_{i}$, let $\hat{F}_{i}^{k}$ be the u.s.c. correspondence from $M$ to $V_{i}$ with nonempty convex (compact) values defined in Claim 3.5. By Cellina [3], for every integer $n$, there exists a continuous mapping $f_{i}^{k, n}: M \rightarrow V_{i}$ such that

$$
G\left(f_{i}^{k, n}\right) \subset G\left(\hat{F}_{i}^{k}\right)+B\left(0, \frac{1}{n}\right) .
$$

Now, for $i \in I$, let $f_{i}^{n}: M \rightarrow\left(V_{i}\right)^{k_{i}}$ be defined as follows:

$$
\forall x \in M, \quad f_{i}^{n}(x)=\left(f_{i}^{1, n}(x), \ldots, f_{i}^{k_{i}, n}(x)\right) .
$$

Applying Theorem 3.1 to the mappings $f_{i}^{n}$, we deduce the existence of $\left(\bar{x}_{i}^{n}\right)_{i \in I} \in M$ such that for every $i \in I, f_{i}^{n}\left(\bar{x}^{n}\right) \in\left(\bar{x}_{i}^{n}\right)^{k_{i}}$, hence

$$
y_{i}^{k, n}:=f_{i}^{k, n}\left(\bar{x}^{n}\right) \in \bar{x}_{i}^{n} .
$$

Since the correspondence $\hat{F}_{i}^{k}$ is bounded ( $M$ is compact and $\hat{F}_{i}^{k}$ is u.s.c.), the sequence $\left(y_{i}^{k, n}\right)$ is bounded. Thus, without any loss of generality, one can suppose that the sequence $\left(y_{i}^{k, n}\right)$ converges to some $y_{i}^{k} \in V_{i}$ when $n$ tends to $+\infty$.

Besides, from the compactness of $M$, without any loss of generality, one can suppose that $\left(\bar{x}_{i}^{n}\right)_{i \in I}$ converges to $\bar{x}=\left(\bar{x}_{i}\right)_{i \in I} \in M$ when $n$ tends to $+\infty$.

Moreover, from Lemma A.1(d) in the appendix and from $y_{i}^{k, n} \in \bar{x}_{i}^{k, n}$, at the limit we have that

$$
\forall i \in I, \forall k=1, \ldots, k_{i}, \quad y_{i}^{k} \in \bar{x}_{i}
$$


Since the graph of $\hat{F}_{i}^{k}$ is closed (it is u.s.c. with compact values) and from $G\left(f_{i}^{k, n}\right) \subset$ $G\left(\hat{F}_{i}^{k}\right)+B(0,1 / n)$, one obtains

$$
y_{i}^{k} \in \hat{F}_{i}^{k}(\bar{x})
$$

To end the proof, we assume that $F_{i}^{k}(\bar{x}) \neq \varnothing$. Since $y_{i}^{k} \in \hat{F}_{i}^{k}(\bar{x})$, by Claim 3.5, there exists $\lambda \in \mathbb{R}$ such that $\lambda y_{i}^{k} \in F_{i}^{k}(\bar{x})$. Hence $\lambda y_{i}^{k} \in F_{i}^{k}(\bar{x}) \cap \bar{x}_{i} \neq \varnothing$ (since $\left.y_{i}^{k} \in \bar{x}_{i}\right)$. This ends the proof of Theorem 2.1.

3.3. Proof of Theorem 2.1 in the general case. We first prove the following lemma.

Lemma 3.6. Let $C$ be a nonempty, convex, compact subset of a Euclidean space $V$. Then there exists a continuous mapping $\rho: G^{1}(V \times \mathbb{R}) \rightarrow C$ such that

$$
\forall x \in G^{1}(V \times \mathbb{R}), \quad x \cap[C \times\{1\}] \neq \varnothing \Longrightarrow x \cap[C \times\{1\}]=\{(\rho(x), 1)\} .
$$

Proof. Since $C$ is compact, it is included in a closed ball $\bar{B}(0, k)$ of $V$. We let $r: V \rightarrow$ $\bar{B}(0, k+1)$ be defined by $r(u)=\alpha(\|u\|) u$, where $\alpha: \mathbb{R}_{+} \rightarrow \mathbb{R}_{+}$is defined by

$$
\begin{aligned}
& \alpha(t)=1 \quad \text { if } t \in[0, k], \\
& \alpha(t)=k+1-t \quad \text { if } t \in[k, k+1], \\
& \alpha(t)=0 \quad \text { if } t \geq k+1 .
\end{aligned}
$$

Let $\pi_{1}: V \times \mathbb{R} \rightarrow V$ and $\rho: G^{1}(V \times \mathbb{R}) \rightarrow C$ be defined by $\pi_{1}(x, t)=x$ and

$$
\rho(x)= \begin{cases}\operatorname{proj}_{C} \circ r \circ \pi_{1}(x \cap[V \times\{1\}]) & \text { if } x \cap[V \times\{1\}] \neq \varnothing, \\ \operatorname{proj}_{C}(0) & \text { if } x \cap[V \times\{1\}]=\varnothing,\end{cases}
$$

where $\operatorname{proj}_{C}: V \rightarrow C$ denotes the projection from $V$ to $C$. Then, one easily sees that $\rho$ satisfies the conclusion of Lemma 3.6.

Proof of Theorem 2.1. Using Lemma 3.6, we first modify the correspondences $F_{j}$ for every $j \in J$ and replace each nonempty compact convex set $C_{j} \subset V_{j}$ by the Grassmannian manifold $G^{1}\left(V_{j} \times \mathbb{R}\right)$. For every $j \in J$, let $\rho_{j}: G^{1}\left(V_{j} \times \mathbb{R}\right) \rightarrow C_{j}$ be the mapping associated to $C_{j} \subset V_{j}$ by Lemma 3.6. Let

$$
\rho: \tilde{M}:=\prod_{i \in I} G^{k_{i}}\left(V_{i}\right) \times \prod_{j \in J} G^{1}\left(V_{j} \times \mathbb{R}\right) \longrightarrow M:=\prod_{i \in I} G^{k_{i}}\left(V_{i}\right) \times \prod_{j \in J} C j
$$

be defined by

$$
\rho(x)=\left(\left(x_{i}\right)_{i \in I}, \rho_{j}\left(x_{j}\right)_{j \in J}\right), \quad \text { for } x=\left(\left(x_{i}\right)_{i \in I},\left(x_{j}\right)_{j \in J}\right) .
$$

For $i \in I$ and $k=1, \ldots, k_{i}$, let $\tilde{F}_{i}^{k}$ be the correspondence from $\tilde{M}$ to $V_{i}$ defined by

$$
\tilde{F}_{i}^{k}(x)=F_{i}^{k}(\rho(x)) \text {. }
$$


For $j \in J$, let $\tilde{F}_{j}$ be the correspondence from $\tilde{M}$ to $V_{j} \times \mathbb{R}$ defined by

$$
\tilde{F}_{j}(x)=F_{j}(\rho(x)) \times\{1\} .
$$

Now, applying the result proved in Section 3.2 (i.e., Theorem 2.1 when $J=\varnothing$ ) to the correspondences $\tilde{F}_{i}^{k}$ and $\tilde{F}_{j}$, there exists $x=\left(\left(x_{i}\right)_{i \in I},\left(x_{j}\right)_{j \in J}\right) \in \tilde{M}$ such that

(i) either $\tilde{F}_{i}^{k}(x) \cap x_{i} \neq \varnothing$ or $\tilde{F}_{i}^{k}(x)=\varnothing$ for every $i \in I$ and $i=1, \ldots, k_{i}$,

(ii) either $\tilde{F}_{j}(x) \cap x_{j} \neq \varnothing$ or $\tilde{F}_{j}(x)=\varnothing$ for every $j \in J$.

Let $\bar{x}=\rho(x) \in M$; we end the proof by showing that it satisfies the conclusion of Theorem 2.1. From the above, it is clearly the case for $i \in I$ and $k=1, \ldots, k_{i}$, that is, we have that

(i) either $F_{i}^{k}(x) \cap x_{i} \neq \varnothing$ or $F_{i}^{k}(x)=\varnothing$ for every $i \in I$ and $i=1, \ldots, k_{i}$.

Now, let $j \in J$. We first notice that $\tilde{F}_{j}(x)=\varnothing$ if and only if $F_{j}(x)=\varnothing$. Assume now that $\tilde{F}_{j}(x) \cap x_{j} \neq \varnothing$ and recall that $x_{j} \cap \tilde{F}_{j}(x)=x_{j} \cap\left(F_{j}(\bar{x}) \times\{1\}\right)$ and $F_{j}(\bar{x}) \subset C_{j}$. Consequently, $x_{j} \cap\left(C_{j} \times\{1\}\right) \neq \varnothing$ and from Lemma 3.6 we get

$$
\varnothing \neq x_{j} \cap\left(F_{j}(\bar{x}) \times\{1\}\right) \subset x_{j} \cap\left(C_{j} \times\{1\}\right)=\left\{\left(\rho_{j}\left(x_{j}\right), 1\right)\right\} .
$$

Hence, the equality holds and $\bar{x}_{j}=\rho_{j}\left(x_{j}\right) \in F_{j}(\bar{x})$. This ends the proof of Theorem 2.1.

\section{Appendix}

\section{The Grassmannian manifold $G^{k}(V)$}

Let $V$ be a Euclidean space and let $k$ be an integer such that $0 \leq k \leq \operatorname{dim} V$. In this section, we recall the properties of $G^{k}(V)$ which are used in this paper.

First, we recall that $G^{k}(V)$ is a smooth boundaryless manifold of dimension $k$ ( $\operatorname{dim} V-$ k) (see, e.g., Hirsch [12] and Lemma A.1). The local charts can be defined as follows. Let $\bar{E} \in G^{k}(V)$ and let $\left\{\bar{e}_{1}, \ldots, \bar{e}_{k}\right\}$ be some given orthonormal basis of $\bar{E}$; we define the mapping $\psi_{\bar{E}}:\left(\bar{E}^{\perp}\right)^{k} \rightarrow G^{k}(V)$ by

$$
\psi_{\bar{E}}(u)=\operatorname{span}\left\{\bar{e}_{1}+u_{1}, \ldots, \bar{e}_{k}+u_{k}\right\}, \quad \text { for } u=\left(u_{1}, \ldots, u_{k}\right) \in\left(\bar{E}^{\perp}\right)^{k} .
$$

Then it is easy to check that the mapping $\psi_{\bar{E}}$ is injective (see Claim A.2); so $\psi_{\bar{E}}$ is a bijection from $\left(\bar{E}^{\perp}\right)^{k}$ onto $U_{\bar{E}}=\psi_{\bar{E}}\left(\left(\bar{E}^{\perp}\right)^{k}\right)$. We can now consider the inverse mapping $\varphi_{\bar{E}}: U_{\bar{E}} \rightarrow\left(\bar{E}^{\perp}\right)^{k}$ defined by $\varphi_{\bar{E}}(E)=\psi_{\bar{E}}^{-1}(E)$, which is clearly a bijection.

Lemma A.1. (a) $G^{k}(V)$ is a smooth boundaryless (i.e., $C^{\infty}$ ) manifold of dimension $k(\operatorname{dim} V-k)$ without boundary and $\left(U_{\bar{E}}, \varphi_{\bar{E}}\right)_{\bar{E} \in G^{k}(V)}$ defines a $C^{\infty}$ atlas of $G^{k}(V)$.

(b) The set $G^{k}(V)$ is compact.

(c) The mapping $E \rightarrow E^{\perp}$ from $G^{k}(V)$ to $G^{\ell}(V)(\ell=\operatorname{dim} V-k)$ is a smooth diffeomorphism.

(d) The mapping $p: V \times G^{k}(V) \rightarrow V$ defined by $p(x, E)=\operatorname{proj}_{E}(x)$ is smooth. Hence, the set $\left\{(x, E) \in V \times G^{k}(V) \mid x \in E\right\}$ is a closed subset of $V \times G^{k}(V)$.

(e) The mapping $x \rightarrow x^{p}$ from $G^{k}(V)$ to $\left(G^{k}(V)\right)^{p}$ is smooth.

We prepare the proof of the lemma with a claim. 
Claim A.2. Let $\bar{E} \in G^{k}(V)$ and let $\left\{\bar{e}_{1}, \ldots, \bar{e}_{k}\right\}$ be an orthonormal basis of $\bar{E}$.

(a) The mapping $\psi_{\bar{E}}$ is injective.

(b) For every $u \in\left(\bar{E}^{\perp}\right)^{k}, \psi(0) \cap \psi(u)^{\perp}=\psi(0)^{\perp} \cap \psi(u)=\{0\}$.

Proof of Claim A.2. Part (a). Let $u=\left(u_{1}, \ldots, u_{k}\right)$ and $v=\left(v_{1}, \ldots, v_{k}\right)$ in $\left(\bar{E}^{\perp}\right)^{k}$ such that

$$
\psi_{\bar{E}}(u)=\operatorname{span}\left\{\bar{e}_{1}+u_{1}, \ldots, \bar{e}_{k}+u_{k}\right\}=\psi_{\bar{E}}(v)=\operatorname{span}\left\{\bar{e}_{1}+v_{1}, \ldots, \bar{e}_{k}+v_{k}\right\} .
$$

Then, there exist some real numbers $\lambda_{i j}(i=1, \ldots, k, j=1, \ldots, k)$ such that

$$
\bar{e}_{i}+u_{i}=\sum_{j=1}^{k} \lambda_{i j}\left(\bar{e}_{j}+v_{j}\right), \quad \text { for every } i=1, \ldots, k
$$

Taking, for each inequality, the scalar product with $\bar{e}_{l}$, where $l=1, \ldots, k$, we obtain $\lambda_{i l}=0$ if $i \neq l$ and $\lambda_{l l}=1$. Hence $u_{l}=v_{l}$ for every $l=1, \ldots, k$, and finally $u=v$.

Part (b). Let $x \in \bar{E} \cap \psi(u)^{\perp}$. Then there exists some real numbers $\lambda_{i}(i=1, \ldots, k)$ such that $x=\sum_{i=1}^{k} \lambda_{i} \bar{e}_{i}$. Taking the scalar product with $\bar{e}_{j}+u_{j}(j=1, \ldots, k)$, we obtain $\lambda_{j}=0$ for every $j=1, \ldots, k$, which proves $\psi(0) \cap \psi(u)^{\perp}=\{0\}$. Similarly, let $x \in \bar{E}^{\perp} \cap \psi(u)$. Then there exists some real numbers $\lambda_{i}(i=1, \ldots, k)$ such that $x=\sum_{i=1}^{k} \lambda_{i}\left(\bar{e}_{i}+u_{i}\right)$. Taking the scalar product with $\bar{e}_{j}(j=1, \ldots, k)$, we obtain $\lambda_{j}=0$ for every $j=1, \ldots, k$, which proves $\psi(0) \cap \psi(u)^{\perp}=\{0\}$ and ends the proof of the claim.

Proof of Lemma A.1. Part (a). We prove that $\left(U_{E}, \varphi_{E}\right)_{E \in G^{k}(V)}$ is a smooth (i.e., $\left.C^{\infty}\right)$ atlas of $G^{k}(V)$ and it is then clear that $\operatorname{dim} M=\operatorname{dim}\left(E^{\perp}\right)^{k}=k(\operatorname{dim} V-k)$. Let $\left(U_{E}, \varphi_{E}\right)$ and $\left(U_{F}, \varphi_{F}\right)$ be two local charts at $E$ and $F$, respectively, such that $U_{E} \cap U_{F} \neq \varnothing$. We will prove that $\varphi_{F} \circ \varphi_{E}^{-1}$ is smooth (i.e., $\left.C^{\infty}\right)$. We let $\left\{e_{1}, \ldots, e_{k}\right\}$ and $\left\{f_{1}, \ldots, f_{k}\right\}$ be two orthonormal bases of $E$ and $F$, respectively. Let $\left(v_{1}, \ldots, v_{k}\right)=\varphi_{F} \circ \varphi_{E}^{-1}\left(u_{1}, \ldots, u_{k}\right)$ and $u=\left(u_{1}, \ldots, u_{k}\right)$; then there exist real numbers $\lambda_{i j}(u)(i, j=1, \ldots, k)$ such that

$$
f_{i}+v_{i}=\sum_{j=1}^{k} \lambda_{i j}(u)\left(e_{i}+u_{i}\right) \quad(i=1, \ldots, k) .
$$

The proof will be complete by showing that the real-valued functions $\lambda_{i j}(u)$ are differentiable with respect to $u$. Taking the scalar product with $f_{l}(l=1, \ldots, k)$, we obtain

$$
f_{i} \cdot f_{l}=\sum_{j=1}^{k} \lambda_{i j}(u)\left(e_{j}+u_{j}\right) \cdot f_{l} \quad(l=1, \ldots, k) .
$$

Thus, for every $i=1, \ldots, k$, the vector $\lambda_{i}(u)=\left(\lambda_{i j}(u)\right)_{j=1}^{k}$ is the solution of a linear system whose matrix $G(u)=\left(\left(e_{j}+u_{j}\right) \cdot f_{l}\right)_{j, l=1, \ldots, k}$ is now shown to be invertible (which clearly implies the differentiability of $\left.\lambda_{i}(u)\right)$. Indeed, if $G(u) \lambda=0$ for some $\lambda \in \mathbb{R}^{k}$, then $\sum_{j=1}^{k} \lambda_{j}\left(e_{j}+u_{j}\right) \cdot f_{l}=0$ (for $\left.l=1, \ldots, k\right)$, thus $\sum_{j=1}^{k} \lambda_{j}\left(e_{j}+u_{j}\right) \in F^{\perp}$. Besides, since $\sum_{j=1}^{k} \lambda_{j}\left(e_{j}+u_{j}\right) \in \psi_{E}\left(u_{1}, \ldots, u_{j}\right)=\psi_{F}\left(v_{1}, \ldots, v_{j}\right)$, we finally obtain $\sum_{j=1}^{k} \lambda_{j}\left(e_{j}+u_{j}\right) \in$ $F^{\perp} \cap \psi_{F}\left(v_{1}, \ldots, v_{k}\right)=\{0\}$ (from Claim A.2). Now, since $\left(e_{j}+u_{j}\right)_{j=1, \ldots, k}$ is a basis, we obtain $\lambda_{j}=0$ for every $j=1, \ldots, k$. 
Part (b). Let $\left(E^{\nu}\right)$ be a sequence in $G^{k}(V)$ and for every $v$, let $\left\{e_{1}^{\nu}, \ldots, e_{k}^{\nu}\right\}$ be an orthonormal basis of $E^{\nu}$. Without any loss of generality, we can assume that for every $i=1, \ldots, k$, the sequence $\left(e_{i}^{v}\right)$ converges to some element $e_{i}$ in $V$. Clearly, $\left\{e_{1}, \ldots, e_{k}\right\}$ is an orthonormal family in $V$, and we let $E=\operatorname{span}\left\{e_{1}, \ldots, e_{k}\right\}$. We will now prove that the sequence $\left(E^{v}\right)$ converges to $E$. Indeed, for $v$ large enough, there exists $u^{v}=\left(u_{1}^{v}, \ldots, u_{k}^{v}\right) \in$ $\left(E^{\perp}\right)^{k}$ such that $E^{v}=\psi_{E}\left(u_{1}^{v}, \ldots, u_{k}^{v}\right)$. It can be written as $e_{i}+u_{i}^{v}=\sum_{j=1}^{k} \lambda_{i j}^{v} e_{j}^{v}(i=1, \ldots, k)$. Multiplying these equalities by $e_{l}(l=1, \ldots, k)$, we obtain $\sum_{j=1}^{k} \lambda_{i j}^{v} e_{j}^{v} \cdot e_{l}=0$ if $i \neq l$ and $\sum_{j=1}^{k} \lambda_{i j}^{v} e_{j}^{v} \cdot e_{i}=1$. This can be written (for every $i=1, \ldots, k$ ) as $\left(e_{j}^{v} \cdot e_{l}\right)_{j, l=1, \ldots, k}\left(\lambda_{i j}^{v}\right)_{j=1}^{k}=$ $\left(e_{i} \cdot e_{l}\right)_{l=1, \ldots, k}$. If $\nu$ is large enough, then $\left(e_{j}^{\nu} \cdot e_{l}\right)_{j, l=1, \ldots, k}$ is invertible and converges to Id, which proves that the sequence $\left(\lambda_{i j}^{v}\right)_{j=1}^{k}$ converges to $\left(e_{i} \cdot e_{l}\right)_{l=1, \ldots, k}$ for every $i=1, \ldots, k$, that is, $\left(\lambda_{i i}^{v}\right)$ converges to 1 and $\left(\lambda_{i j}^{v}\right)$ converges to 0 for $i \neq j$. We finally obtain that $\left(u_{i}^{v}\right)$ converges to 0 , which proves that $E^{v}$ converges to $E$.

Part (c). Let $\bar{E} \in G^{k}(V)$ and let $\left(\bar{e}_{1}, \ldots, \bar{e}_{k}\right)$ and $\left(\bar{f}_{1}, \ldots, \bar{f}_{\ell}\right)$ be orthonormal bases of $\bar{E}$ and $\bar{E}^{\perp}$, respectively. Let $\left(u_{1}, \ldots, u_{k}\right) \in\left(\bar{E}^{\perp}\right)^{k}$ and let $E=\psi(u)$. Then it is easy to see that $E^{\perp}=\operatorname{span}\left\{f_{1}+v_{1}, \ldots, f_{\ell}+v_{\ell}\right\}$, where $v_{i}=-\sum_{j=1}^{k}\left(f_{i} \cdot u_{j}\right) \bar{e}_{j}$. So each $v_{i}$ is a smooth mapping with respect to the $u_{i}$ and, conversely, from $\left(E^{\perp}\right)^{\perp}=E$, each $u_{i}$ is a smooth mapping with respect to the $v_{i}$. This ends the proof of part (c).

Part (d). The differentiability of the mapping $p$ is left to the reader. Then notice that $\left\{(x, E) \in V \times G^{k}(V) \mid x \in E\right\}=\left\{(x, E) \in V \times G^{k}(V) \mid x=\operatorname{proj}_{E}(x)\right\}$, which is clearly closed since the mapping $p:(x, E) \rightarrow \operatorname{proj}_{E}(x)$ is continuous.

\section{References}

[1] P. Bich and B. Cornet, Existence of financial equilibria: space of transfers of fixed dimension, Tech. Report, Université de Paris 1, Paris, 1998.

[2] L. Brouwer, Über Abbildung von Mannigfaltigkeiten, Math. Ann. 71 (1911), 97-115 (German).

[3] A. Cellina, Approximation of set valued functions and fixed point theorems, Ann. Mat. Pura Appl. (4) 82 (1969), 17-24.

[4] - A theorem on the approximation of compact multivalued mappings, Atti Accad. Naz. Lincei Rend. Cl. Sci. Fis. Mat. Natur. (8) 47 (1969), 429-433.

[5] B. Doubrovine, S. Novikov, and A. Fomenko, Géométrie Contemporaine. Méthodes et Applications. II. [Modern Geometry. Methods and Applications. II], "Mir", Moscow, 1982.

[6] D. Duffie and W. Shafer, Equilibrium in incomplete markets. I. A basic model of generic existence, J. Math. Econom. 14 (1985), no. 3, 285-300.

[7] S. Eilenberg and D. Montgomery, Fixed point theorems for multi-valued transformations, Amer. J. Math. 68 (1946), 214-222.

[8] D. Gale and A. Mas-Colell, An equilibrium existence theorem for a general model without ordered preferences, J. Math. Econom. 2 (1975), no. 1, 9-15.

[9] P. Gourdel, Existence of intransitive equilibria in nonconvex economies, Set-Valued Anal. 3 (1995), no. 4, 307-337.

[10] V. Guillemin and A. Pollack, Differential Topology, Prentice-Hall, New Jersey, 1974.

[11] M. Hirsch, M. Magill, and A. Mas-Colell, A geometric approach to a class of equilibrium existence theorems, J. Math. Econom. 19 (1990), no. 1-2, 95-106.

[12] M. W. Hirsch, Differential Topology, Springer-Verlag, New York, 1976.

[13] S. Y. Husseini, J.-M. Lasry, and M. Magill, Existence of equilibrium with incomplete markets, J. Math. Econom. 19 (1990), no. 1-2, 39-67.

[14] S. Kakutani, A generalization of Brouwer's fixed point theorem, Duke Math. J. 8 (1941), 457-459. 
[15] E. Michael, Continuous selections. I, Ann. of Math. (2) 63 (1956), 361-382.

Philippe Bich: Centre de Recherche en Mathématiques de la Décision, Université Paris-Dauphine, Place du Maréchal de Lattre de Tassigny, 75775 Paris, France

E-mail address: bich@ceremade.dauphine.fr

Bernard Cornet: Centre de Recherche en Mathématiques, Statistique et Economie Mathématique, Université Paris 1, 106-112 boulevard de l'Hôpital, 75647 Paris, France

E-mail address: cornet@univ-paris1.fr 